\title{
ARE CREWS EMPOWERED WITH ALL THE RESOURCES NEEDED TO SUCCESSFULLY ADDRESS AN INFLIGHT EMERGENCY? CHECKLISTS, A NECESSARY BUT INSUFFICIENT TOOL
}

\author{
Daniel MUÑOZ-MARRÓN (iD ${ }^{1^{*}}$, Francisco GIL (i) ${ }^{2}$, Ana LANERO (iD ${ }^{3}$ \\ ${ }^{1} 43^{\text {rd }}$ Air Force Group, Spanish Air Force, Torrejón de Ardoz, Spain \\ ${ }^{2}$ Faculty of Psychology, Complutense University of Madrid, Madrid, Spain \\ ${ }^{3}$ Faculty of Economy, University of León, León, Spain
}

Received 17 August 2018; accepted 20 September 2018

\begin{abstract}
Although safety in aviation has increased exponentially in recent decades, sadly, plane crashes will always be associated with the operation of the aviation world. The efforts should be directed towards the pursuit of "zero accidents", providing aircrews with the necessary resources to minimize risks. One of the most important flight operation resources used in aviation is the checklist. However, when faced with an emergency, the crew cannot always make use of them, either because the huge diversity of potential emergencies does not allow to collect all of them in these manuals, or because the time available to react is so short that it makes it impossible to utilize them. This paper shows how aircraft checklists are a necessary but insufficient tool to solve complex, unpredictable, and novel emergencies. Furthermore, based on successfully solved critical events, the authors suggest the implementation of specific techniques for the learning and development of problem-solving programs focused on innovation and divergent thinking to decision making, as part of flight training, in order to increase the crews' capability to face such emergencies.
\end{abstract}

Keywords: aviation psychology, aviation safety, checklist, creativity, crew resource management (CRM), divergent thinking, flight crew training, military aviation, teamwork.

\section{Introduction}

Why do aviation accidents occur? What are their causes? Can they be avoided or at least their occurrence minimized? Since the Wright brothers made the first flight in history with their Flyer I in Kitty Hawk in 1903, aviation has advanced remarkably fast throughout its barely more than 115 years of history. Aeronautical engineering has evolved considerably, both in the construction of aircraft as well as in the devices and navigation systems that they carry on board. This has made the modern flight so automated that it seems to have reduced the workload of the crew to a minimum. However, this statement is far from reality. No machine, including state-of-the-art aircraft, can do all the work on its own, much less when unforeseen situations arise, leaving it to human aircrews to execute, control or supervise the machine. In addition, research shows that most air accidents are caused by human error (Helmreich, Merritt, \& Wilhelm, 1999). Hence, the increasing importance given to the human aspects related to flight by all public and private entities in aviation (International Air Transport Association [IATA], 2015).

Aircraft technology allows flight professionals to virtually become "machine supervisors". However, their decision-making remains critical, even more when they face critical situations that technology cannot anticipate or forecast, and which, therefore, the machine is not able to solve on its own. Certain measures can be established to minimize errors, and protocols, known in the world of aviation as checklists, are one of the most useful. Protocols help to ensure that everything that can be controlled is controlled, going so far as to make aviation one of the work areas in which the protocols of action are the most standardized, and, thus, serving as a model for other fields, such as medicine, nuclear plants or crisis management. In the case of the medical sector, more and more people are working to save lives and improve patient care by using the airline industry's advances and knowledge on safety and teamwork (Gordon, Mendenhall, \& O'Connor, 2013).

*Corresponding author. E-mail: danmun01@ucm.es 
The importance of checklist use as a function of the treatment contingency has been addressed by different authors (Rantz, Dickinson, Sinclair, \& Van Houten, 2009). Some of this previous research has focused on checklist design and composition (Degani \& Wiener, 1990, 1997; Heymann, Degani, \& Barshi, 2007), while other studies have addressed checklist compliance (Helmreich, Klinect, Wilhelm, \& Jones, 1999; Helmreich, Wilhelm, Klinect, \& Merritt, 2001; Klinect, Murray, Merritt, \& Helmreich, 2003), the challenge of memorized procedures (Pettitt \& Dunlap, 1997) or its completion in a precise way. What is clear is that procedures represent the best way to achieve safe operations (Wieringa, Moore, \& Barnes, 1992) and to assist the crew along a path of pre-defined sequences of actions (Degani, Heymann, \& Shafto, 1999).

Pilot discipline leads them to use these protocols as part of their performance of standardized procedures (Sculli \& Sine, 2011), which are quite complete for dealing with routine tasks or hazards that have already occurred. However, it must be recognized that an unpredictable constellation of circumstances (including machine, human and environmental factors) can interfere with flight operations (Mosier, Palmer, \& Degani, 1992). The potential incidents that can happen in flight are infinite and, consequently, the protocols of action provided to operators are insufficient to cope with each and every one of the unforeseen events.

This paper seeks to advance this line of research by analysing the potential ways to improve cockpit teamwork, after demonstrating that checklists are insufficient to cope with certain air emergencies. We argue that checklists are unable to include the thousands of new or combined situations that may arise, and that, in many air emergency situations, the limitation of the time of action, "a race against time" (Heymann et al., 2007), is a fundamental factor that prevents the use of such checklists. It is well known that the impact with a passing flock of geese motivated the successful forced landing of US Airways Flight 1549 on the Hudson River (New York, US) in January 2009; or that the sudden changes in atmospheric conditions led to the crash of Air France Flight 447 in the middle of the Atlantic Ocean, causing the death to 228 souls in June of that year; or that the causes which are yet to be clarified led the A400-M to crash in the vicinity of the San Pablo airport in Seville (Spain) in May 2015. These are real examples of situations in which the use of checklists could hardly have prevented the accident.

Within this framework, the research goal of this study is to show that a great number of flight emergency situations are not collected in a checklist. Therefore, some of the most unpredictable aerial accidents that take place in flight cannot be solved simply by the use of the aircraft's operational checklists. The paper is structured as follows. Firstly, we review the previous literature on operational procedures and checklists. Next, the methodology section describes the data sample and procedure used to collect the data of the study. Afterwards, we present the results and discuss their practical implications, introducing one main idea: the development of problem-solving and decision-making creative thinking programs, as part of flight crew training, which may be the key aspects of human factor that can help to overcome such limiting situations.

\section{Are all emergencies collected in checklists?}

Checklists are a basic element in the airline industry, to the point of being considered by some authors as the backbone of the cabin safety culture (Sculli \& Sine, 2011). The use of checklists is a technique introduced in the first decades of the twentieth century, and currently used by all civilian and military operators in the world. First they adopted the form of paper checklists, which later evolved to electronic ones. Nowadays, there are two fundamental types of checklists: read-and-verify checklists, used for routine procedures to verify that "everything is as it should be", and read-and-do checklists, used to solve unusual situations that require performance by the crew. The first ones (also known as normal checklists) serve to confirm or correct the configuration of the aircraft facing critical events once the corresponding Standard Operating Procedures (SOPs) have been performed (Airbus, 2004). The correct completion of normal checklists is essential for the safe execution of procedures during all phases of the flight, in particular during the take off, approach and landing maneuvers.

Conversely, the read-and-do checklists include those actions that crews must perform (some as memorized items and others as read items) in a particular airplane model, step by step, for an endless number of emergencies, critical events or actions not usually foreseen. Both the variety and the number of emergencies that can occur in an aircraft are practically unlimited, and this is precisely what leads us to defend that it is impossible that the readand-do checklist of each aircraft model contains all the possible events that the crews can come across.

The advances of aeronautical engineering have been of such magnitude that it is almost impossible that a certain event, one of those considered "usual" (and therefore included in the checklists) will be able to bring down an airplane, leaving it for the most part to the occurrence of extraordinary events that will lead to appearance of a potential danger of real risk. Under threatening conditions, crews will inevitably attempt to fly following the checklist (Helmreich, 1999), and it is true that one of the contributions of the sixth generation of Crew Resource Management (CRM), known as threat and error management (Helmreich, 2006; Marshall, 2010), is precisely that these procedures are a critical means of trapping and mitigating errors and threats. However, what happens when the emergency that is presented is not detailed in the aircraft's operating procedures, or the reaction time needed does not allow consulting them? When one of these "unregistered" events occurs, crews 
find themselves without tools, helpless in the face of an emergency situation for which they do not have any manual to refer to. Furthermore, their training has been focused on very specific situations and actions in which the protocol must be followed in a strict and disciplined way (it is true that military pilots, more than civil ones, receive certain training to face critical "unregistered" events since Air Force Academies usually include them in their flight training courses). When facing an unregistered event, the pilot must rely on his or her judgment to decide which list to use (Sine \& Sculli, 2010), but they do not always have a list to use.

By simply looking at some of the most significant accidents suffered by airlines in the brief, but exciting, aviation history, we can find countless instances which were not detailed in the aircraft's checklist of operation. Does this mean that checklists are not useful in resolving cockpit situations? The answer is that they are really useful indeed. The checklist helps to avoid the occurrence of errors caused by memory failures (Norman, 1990), supports the situational awareness, making the attention go to the right place at the right time (Sculli \& Sine, 2011) and facilitates the management of workload. In all high-risk industries, the procedures supplied to the operators provide the best way to perform a task (Degani et al., 1999). So, checklists are adequate tools when operating an aircraft, but fundamentally for those more routine and standardized tasks. However, on their own, they are not sufficient to take care of all the situations, primarily the emergency ones, and more specifically those entailing high risk, precisely because usually such situations cannot be foreseen. The next section describes the methodology of an empirical study carried out to demonstrate this assertion and identify alternative ways to improve the functioning of cockpit procedures.

\section{Hypotheses}

The checklist is a significant tool in aviation (Sculli \& Sine, 2011; Young-Xu et al., 2013) as well as in other areas (Gordon et al., 2013; Sculli \& Sine, 2011). However, it is impossible to include all possible air incidents and emergencies in them, particularly the most unusual, unpredictable and dangerous ones, hence, the crews are not able to solve all the air emergencies using checklists. In addition, the problem is agravated by the fact that flight-crew members are not always aware of that. This leads us to propose the following hypotheses.

Hypothesis 1. A large number of emergencies that may occur during a flight are not listed in the aircraft checklists.

Hypothesis 2. Most emergencies that crews consider to be the most dangerous are precisely those that are not included in the checklists.

Hypothesis 3. A high percentage of flight-crew members are unaware that most emergencies that occur during a flight are not listed in their aircraft checklists.

\section{Method}

Because of the irrefutable fact that there are numerous air emergencies which are impossible to collect in the aircraft's SOPs and checklists, the objective of the present research is to reveal what percentage of emergency situations that arise during a flight are not included in the checklists. To do that, we conducted a two-tasks survey study, which is described in the next sections.

\section{Participants}

To collect the data of the study, we asked the $43^{\text {rd }}$ Air Force Group of the Spanish Air Force to participate. This Air Force Unit is dedicated to aerial firefighting and search and rescue missions flying amphibious aircraft Canadair CL-215T and Bombardier CL-415, and has its headquarter in the Torrejón Air Force Base (Madrid, Spain). Although this Unit was selected for convenience, it can be considered representative of other crews facing crisis situations and unpredictable events and, according to the research purposes, allows investigating how teams cope with complex problems, adapt to changing demands, and learn from their actions.

Two different sample categories were selected for each of the two research tasks described below. However, due to the limited universe of the sampling, some of the components of sample 1 were also part of sample 2 (specifically, 8 people). There are very few pilots in Spain who operate this type of aircraft and the $43^{\text {rd }}$ Air Force Group includes most of the members of this small group of professionals. While it is true that the INAER company was flying a small number of such aircraft until 2014 also with civilian pilots, currently only the $43^{\text {rd }}$ Air Force Group operates this type of aircraft in Spain.

Sample 1. Sample 1 was composed of 19 flight crews from Canadair CL-215T/Bombardier CL-415 amphibious aircraft. The standard crew of the $43^{\text {rd }}$ Group consists of three members: a Pilot in Command (PIC), a First Officer (FO) and a Flight Engineer (FE). The sample consisted of a total of 57 people of both sexes, 38 aircraft pilots (19 PIC and 19 FO) and 19 Flight Engineers, all of them officers and non-commissioned officers from the Spanish Air Force, who performed the first task of the study during their weekly flight simulator training. Three pilots who participated in the pilot study to create the questionnaires for a broader study had to be removed from the 57 participants of the initial sample, so Sample 1 finally included a total of 54 crewmembers (35 pilots and 19 flight engineers).

Sample 2. To address the second task, it was considered appropriate to use a more selective sample, choosing exclusively those professionals with more knowledge and experience in this type of aircraft and its mode of operation. To this end, 10 pilots (PIC) and 3 flight engineers with Type Rating Instructor (TRI) and Maintenance Test Pilot/Engineer category were selected. This category 
represents the highest training level within the operation of the Canadair CL-215T/Bombardier CL-415, making them a group of experts with a very high knowledge on the subject.

\section{Procedure}

To test the research hypotheses, we carried out an empirical study aimed at analyzing the extent to which checklists include the air emergencies identified as the most dangerous by professional experts. The study was part of larger experimental research on aviation safety and consisted of two different survey tasks, performed, respectively, by the samples 1 and 2 described in the previous section. In each case, the participants were asked to answer a paper questionnaire in a room usually used for pre and post briefing during the simulator crew training in the $43^{\text {rd }}$ Air Force Group facilities. To control for social desirability bias, participants were requested to respond to the questionnaire as honestly as possible, and confidentiality of answers was ensured.

First Task. Participants of sample 1 answered the first questionnaire during a pre-briefing before a routine biannual training session. The questionnaire included the following question: "List those incidents or accidents (if any) that you have encountered and consider important since you started to operate in the $43^{\text {rd }}$ Air Force Group. Define or identify each situation and assign to each one a value on a scale (from $0=$ little risk situation, to $10=$ very high risk situation)".

All participants, pilots and flight engineers, had to briefly report the most dangerous situations they had experienced as flight crew since their arrival to this Unit of the Spanish Air Force. They also had to assess the severity of each situation mentioned, according to an 11-point Likert-type scale. This procedure had a double purpose. Firstly, to identify the most dangerous events overcome by the crewmembers during their flights. Secondly, to check what percentage of the total situations reported and identified as dangerous were included in the checklists of the aircraft.

Second Task. To avoid associations and interferences with the first task, sample 2 was presented with a second questionnaire six months later, during the Flight Safety Working Day that the $43^{\text {rd }}$ Air Force Group holds twice a year. This working day is a day exclusively dedicated to air safety. All the members of the Unit (head staff, pilots, flight engineers and maintenance people) work together sharing experiences in order to improve air safety standards of the Unit through the analysis of accidents, discussion groups, videos, role-playing, conferences, etc., focusing on crew resource management (CRM) and key aspects of human factors applicable to aviation (MuñozMarrón, 2018). This time, the participants were asked to assess the assertion: "the emergencies that can be presented by this airplane are included in the checklists", according to a 10-point Likert-type scale ranging from 0 (not at all) to 9 (totally).
The purpose in this case was to get a firsthand opinion of the crews about the false beliefs about their checklists. Once the most dangerous emergencies were identified and their inclusion in the checklists of Canadiar CL-215T/ Bombardier CL-415 was checked (first task), the data collected in this second questionnaire was used to find out whether the crewmembers were aware of this limitation or not, and therefore, to analyze the actual usefulness of existing operational checklists to cope with extreme airborne emergencies.

\section{Results}

The participants of Sample 1 reported a total of 118 emergency situations. Some examples of these situations were: "crossing another aircraft in flight", "main gear puncture during landing", "flying into the smoke of a wildfire while approaching to drop the water", "climbing to high altitude without oxygen supply in order to avoid bad weather", "impact with electric lines", "bird strike", "stalling in a turn", "encountering descending wind-shear near the ground", "disorientation with vertigo", "near collision with other airborne means operating in a fire", "elevator trim failure during take off", "icing during cruise", "nose wheel blowout", "loss of power in the airplane with associated loss of lift", "loss of both engines due to smoke intake", "severed engine power control cables associated to engine \#1", "short circuit in the flap system", and so on.

However, only 15 out of these 118 reported situations appeared in the Canadair CL-215T/Bombardier CL-415 amphibious checklist (the checklists of both aircraft are quite similar). This implies that only $12.71 \%$ of the events reported by the crews participating in the study (for example, "total loss of hydraulic fluid", "engine fire on the ground" or "water door failure") have a solution procedure through the application of the checklist. This leaves the rest of the emergency situations, an alarming $87.29 \%$, without a standardized solution procedure. This result supports the first hypothesis.

Regarding Hypothesis 2, we analysed the scores reported by pilots and flight engineers of Sample 1 when they were asked to assess the potential risk of the emergency situations identified. These results are summarized in Table 1 and Table 2.

It is interesting that only 21 of the 118 situations reported $(17.79 \%)$ were scored under the intermediate value of 5 in a 0 to 10 scale. This might be due to the fact that, when the crews describe emergency situations, they usually remember the most dangerous ones, thus assessing them with high values. Similarly, the median $\left(50^{\text {th }}\right.$ percentile) and the mode, which show the distribution of the scores, corresponded to the values of 7 and 8 , respectively.

In order to analyze the second hypothesis, Figure 1 illustrates this data to clarify that the most dangerous situations (those with values 7 and above according to the scores given by the members of Sample 1) are precisely those not included in the checklists. 
Table 1. Scores given by the members of Sample 1 to the dangerous situations that they have encountered during their aeronautical career in the $43^{\text {rd }}$ Air Force Group

\begin{tabular}{|c|c|c|c|c|}
\hline Scores & $\begin{array}{c}\text { Number of dangerous } \\
\text { situations for each score }\end{array}$ & Percentage $\%$ & $\begin{array}{c}\text { Cumulative number of } \\
\text { dangerous situations }\end{array}$ & $\begin{array}{c}\text { Cumulative } \\
\text { percentage } \%\end{array}$ \\
\hline 0 & 2 & 1.69 & 2 & 1.69 \\
\hline 1 & 2 & 1.69 & 4 & 3.38 \\
\hline 2 & 4 & 3.38 & 8 & 14.77 \\
\hline 3 & 6 & 5.08 & 21 & 17.79 \\
\hline 4 & 7 & 5.93 & 35 & 29.66 \\
\hline 5 & 14 & 11.86 & 45 & 38.13 \\
\hline 6 & 10 & 8.47 & 65 & 55.08 \\
\hline 7 & 20 & 16.94 & 88 & 74.57 \\
\hline 8 & 23 & 19.49 & 104 & 88.13 \\
\hline 9 & 16 & 13.55 & 118 & 100 \\
\hline TOTAL & 14 & 11.86 & - & - \\
\hline
\end{tabular}

Table 2. Emergency situations described by Sample 1 which were included vs. not included in the aircraft operation checklists

\begin{tabular}{|c|c|c|c|c|c|c|c|}
\hline & & \multicolumn{4}{|c|}{ Emergencies reported } & \multirow{2}{*}{\multicolumn{2}{|c|}{ TOTAL }} \\
\hline & & \multicolumn{2}{|c|}{ Included } & \multicolumn{2}{|c|}{ Not included } & & \\
\hline & & $\mathrm{N}$ & $\%$ & $\mathrm{~N}$ & $\%$ & $\mathrm{~N}$ & $\%$ \\
\hline \multirow{11}{*}{$\begin{array}{l}\text { Scores in the } \\
\text { danger scale }\end{array}$} & 0 & 0 & 0 & 2 & 1.69 & 2 & 1.69 \\
\hline & 1 & 1 & 0.84 & 1 & 0.84 & 2 & 1.69 \\
\hline & 2 & 1 & 0.84 & 3 & 2.54 & 4 & 3.38 \\
\hline & 3 & 2 & 1.69 & 4 & 3.38 & 6 & 5.08 \\
\hline & 4 & 2 & 1.69 & 5 & 4.23 & 7 & 5.93 \\
\hline & 5 & 5 & 4.23 & 9 & 7.62 & 14 & 11.86 \\
\hline & 6 & 2 & 1.69 & 8 & 6.77 & 10 & 8.47 \\
\hline & 7 & 1 & 0.84 & 19 & 16.10 & 20 & 16.94 \\
\hline & 8 & 0 & 0 & 23 & 19.49 & 23 & 19.49 \\
\hline & 9 & 1 & 0.84 & 15 & 12.71 & 16 & 13.55 \\
\hline & 10 & 0 & 0 & 14 & 11.86 & 14 & 11.86 \\
\hline \multicolumn{2}{|c|}{ TOTAL } & 15 & 12.71 & 103 & 87.28 & 118 & 100 \\
\hline
\end{tabular}

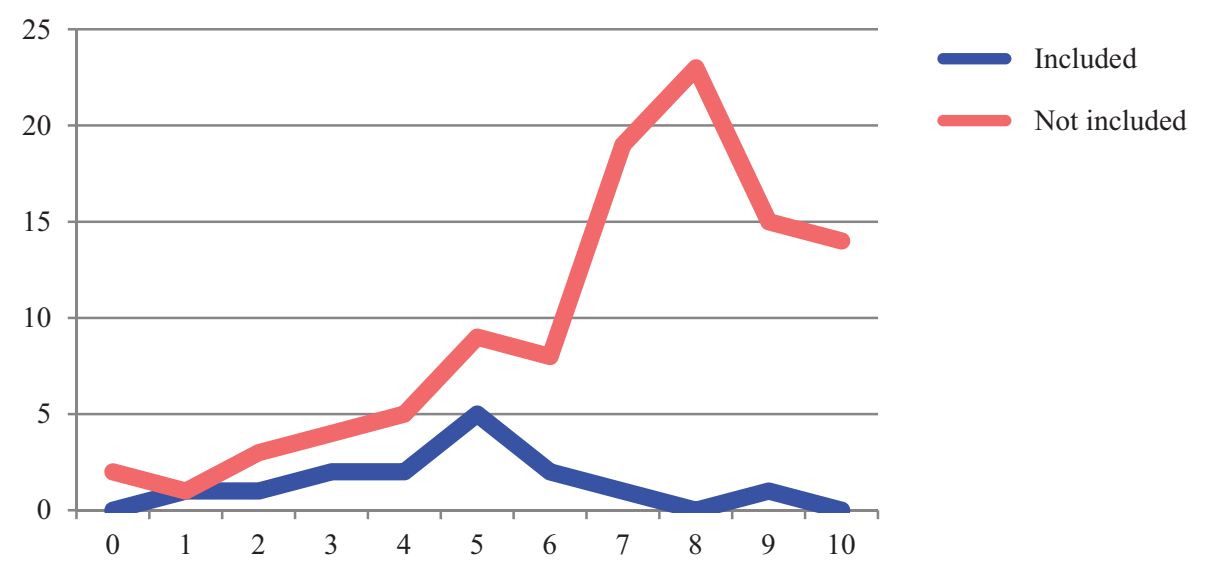

Figure 1. Graphic representation of emergency situations included vs. not included in checklists 
Additionally, to test this hypothesis, we performed a Student's $t$-distribution for independent samples, comparing the mean score given to situations included and not included in the checklist. The results supported the existence of statistically significant differences between the two groups ( $t=3.68, \mathrm{p}<.005)$, so that emergencies not included in the checklist obtained a mean of $7.03(S D=2.37)$ in the scale of danger, much higher than the mean score of 4.67 (SD = 1.99) obtained for the situations included. This result provides initial support to Hypothesis 2 .

Delving into these results, we performed two additional analyses to examine the percentage of situations perceived as extremely dangerous that are included in the checklists. For the first analysis, we selected the situations with the danger scores above the $50^{\text {th }}$ percentile (exactly above the $55^{\text {th }}$ percentile) while in the second one, we used the data from the last quartile, filtering from the situations evaluated as dangerous, those that were so to a maximum extent. From the total (see Table 1), 53 situations scored higher than the $56^{\text {th }}$ percentile (values of 8 or higher), while 30 situations were included in the last quartile (values of 9 or 10). Table 3 presents the results of the first contingency analysis performed to compare the percentage of the 53 most dangerous situations that were included versus not included in the aircraft checklist. Meanwhile, Table 4 shows the results of the second contingency analysis on the 30 dangerous situations of the last quartile.

As expected, the first analysis revealed that only one (1.89\%) of the 53 situations was included in the checklist, and therefore, had a standardized resolution procedure (specifically, "elevator trim failure during take off"). The remaining reported situations, that is 52 (98.11\%) of those considered extremely dangerous, did not appear on the aircraft's operational checklists (see Table 3). Furthermore, according to the second analysis (see Table 4), this was the only situation of the 30 most dangerous situations included in the checklist $(3.33 \%)$. These results support Hypothesis 2.

Finally, in order to test the third hypothesis, the data provided by the members of Sample 2, that is the group of 13 experts, regarding the degree to which the emergencies are included in the checklist, was used. As described before, this group of experts had to assign an individual score to this question, based on their experience. The results obtained are presented in Table 5.

Table 3. Contingency analysis results for the emergency situations above the $55^{\text {th }}$ percentile

\begin{tabular}{|c|c|c|c|c|c|c|c|}
\hline & & \multicolumn{4}{|c|}{ Emergencies reported } & \multirow{2}{*}{\multicolumn{2}{|c|}{ TOTAL }} \\
\hline & & \multicolumn{2}{|c|}{ Included } & \multicolumn{2}{|c|}{ Not included } & & \\
\hline & & $\mathrm{N}$ & $\%$ & $\mathrm{~N}$ & $\%$ & $\mathrm{~N}$ & $\%$ \\
\hline \multirow{3}{*}{$\begin{array}{l}\text { Scores in a } \\
\text { danger scale }\end{array}$} & 8 & 0 & 0 & 23 & 43.39 & 23 & 43.39 \\
\hline & 9 & 1 & 1.89 & 15 & 28.3 & 16 & 30.19 \\
\hline & 10 & 0 & 0 & 14 & 26.42 & 14 & 26.42 \\
\hline \multicolumn{2}{|c|}{ TOTAL } & 1 & 1.89 & 52 & 98.11 & 53 & 100 \\
\hline
\end{tabular}

Table 4. Contingency analysis results for the emergency situations of the last quartile

\begin{tabular}{|c|c|c|c|c|c|c|c|}
\hline & & \multicolumn{4}{|c|}{ Emergencies reported } & \multirow{2}{*}{\multicolumn{2}{|c|}{ TOTAL }} \\
\hline & & \multicolumn{2}{|c|}{ Included } & \multicolumn{2}{|c|}{ Not included } & & \\
\hline & & $\mathrm{N}$ & $\%$ & $\mathrm{~N}$ & $\%$ & $\mathrm{~N}$ & $\%$ \\
\hline \multirow{2}{*}{$\begin{array}{l}\text { Scores in a } \\
\text { danger scale }\end{array}$} & 9 & 1 & 3.33 & 15 & 50 & 16 & 53.33 \\
\hline & 10 & 0 & 0 & 14 & 46.67 & 14 & 46.67 \\
\hline \multicolumn{2}{|c|}{ TOTAL } & 1 & 3.33 & 30 & 96.67 & 30 & 100 \\
\hline
\end{tabular}

Table 5. Scores assigned to the second task by the group of experts from Sample 2

\begin{tabular}{|c|c|c|c|c|c|c|c|c|c|c|c|c|c|}
\hline Expert number & 1 & 2 & 3 & 4 & 5 & 6 & 7 & 8 & 9 & 10 & 11 & 12 & 13 \\
\hline Position in the crew & \multicolumn{10}{|c|}{ PIC (Pilot in Command) } & \multicolumn{3}{|c|}{ Flight Engineer } \\
\hline $\begin{array}{l}\text { Professional category } \\
\text { for Canadair CL-215T / } \\
\text { Bombardier CL-415 }\end{array}$ & 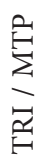 & 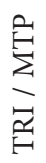 & 买 & $\stackrel{\text { 空 }}{\Sigma}$ & 止 & 疋 & 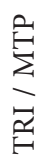 & 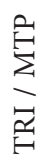 & 巫 & 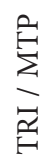 & 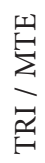 & 点 & 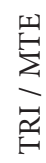 \\
\hline Score & 7 & 7 & 6 & 8 & 9 & 8 & 8 & 7 & 2 & 8 & 8 & 8 & 7 \\
\hline \multicolumn{12}{|c|}{ Mean } & \multicolumn{2}{|c|}{7.15} \\
\hline
\end{tabular}


According to the results, almost all experts, 12 out of $13(92.30 \%)$, assigned a score of 6 or higher to the question posed on a 0 to 9 scale, which means that they think their checklists contain most of the emergencies that can occur during the flight. Specifically, the total sample obtained a mean score of 7.15 , with a median and mode of 8. This finding points to a biased perception of the participants, as, according to our previous results, most emergency situations identified did not appear in the checklist (Hypothesis 1). Therefore, Hypothesis 3 is also supported.

\section{Conclusions}

The analysis of human error allows us to examine a wide range of causes that may lie behind an accident (Reason, 1990). Thus, these results show a very specific reality related to the world of aviation, focused on the following conclusions which the aeronautical industry should take into account and that future research on aviation safety should keep in mind.

A large number of air emergencies occurring on airplanes do not have written resolution procedures. Possibly, this fact is related to the impossibility of collecting all of them in a checklist due to the amount of situations they imply and, especially, the huge variety of factors and variables that influence their occurrence and make them unpredictable.

One of the most intriguing findings of the present study supporting such reasoning points to the general conclusion that, according to the view of crews, the most dangerous situations that can occur during a flight are those that are not detailed in a checklist. Therefore, some of the most unpredictable aerial accidents that take place in flight cannot be solved simply by the use of the aircraft's operational checklists. Furthermore, the results of the research suggest that crews do not seem to be aware that the checklists they use have this limitation and, consequently, have insufficient resources to address certain dangerous situations.

These results are not intended to question the elaboration and design of checklists in aviation. Checklists are indeed a useful resource, the design of which serves as a model for other scientific fields (Sculli \& Sine, 2011). They remain a vital component of safe operations in the flight environment (Rantz et al., 2009) and a fundamental tool for crews to manage air emergencies. However, this research reveals an aspect that has passed relatively unnoticed in the previous literature. Certainly, situations such as a near miss with another aircraft in flight, an impact with an electric wire or a bird, the stall of the airplane during a turn, severed engine power control cables or a short circuit in the flap system, to quote some of the situations reported by the crews, are really difficult to include in a checklist. Also, it must be added that the crews are not aware of the limitations that these checklists present.

Emergency procedures always involve the sudden appearance of a degraded condition with an imminent path to a catastrophe and the existence of measured steps to prevent it (Heymann et al., 2007); however, crews do not have procedures for every real emergency. Therefore, other complementary mechanisms that give crews sufficient resources to deal successfully with this type of emergency situations are absolutely indispensable. At the same time, it becomes necessary for the crews to realize that the currently available resources are not perfect and can be improved in order to achieve success in all air operations as well as the long awaited utopian "zero accident rate".

In line with this reasoning, creativity in a teamwork context refers to the processes by which the members of a team generate novel and useful ideas to solve problems (Hennessey \& Amabile, 2010; Zhou \& Hoever, 2014) as part of the decision-making process. In most emergency incidents, included the ones identified by the sample of this study, crews' performance becomes a critical factor. This performance needs to use some elements of creative thinking, especially in critical situations, in order to take a brilliant decision to solve them. Thus, the aviation industry should provide crews with specific training in flexibility and divergent thinking to improve their human factors training. Crews must continue using the checklists for all those routine and emergency situations that allow their use, but such complementary training will help them to make quick and effective decisions in those novel and unpredictable situations impossible to solve by using checklists.

Additionally, it is important to complement crew training with workout programs that prepare them to face these highly dangerous events. It is essential to include some aspects focused on innovation and creative problem solving into the crew training programs, which, according to empirical facts (like, among many others, the accidents mentioned in the Introduction), have made it formerly possible for a crew to solve an emergency that appeared to be unequivocally leading to a disaster. It is possible to improve the coordination training of aircrews with syllabuses that include tools for searching for creative solutions under time constraints. Specific programs and techniques to develop creativity, flexibility and divergent thinking in decision making would maximize the aptitudes and skills of the crews (Muñoz-Adánez, 2006), thus allowing them to deal with complex unforeseeable events for which there is no standardized written procedure.

The benefits of flexible team interactions during critical situations have already been featured by Stachowski, Kaplan, and Waller (2009) in a study simulating nuclear crisis to observe the adherence of the crisis management team to standard procedures, as opposed to the tendency to move away from them when facing unexpected events. The training programs of the crews can be improved by including this key aspect, thus providing airline companies and Air Force Units around the world with a valuable tool to deal with those crisis situations that can be fatal in a flight.

The present study is framed by the previous literature on supporting factors to team adaptability and effectiveness (Burke, Stagl, Salas, Pierce, \& Kendall, 2006; Dietz et al., 2017) and tries to contribute to the research about 
design (Degani et al., 1999; Degani \& Wiener, 1990, 1997; Heymann et al., 2007) and utility (Helmreich, 1999; Helmreich, Klinect et al. 1999, Helmreich et al., 2001; Klinect et al., 2003; Pettitt \& Dunlap, 1997; Rantz et al., 2009; Sculli \& Sine, 2011; Sine \& Sculli, 2010) of checklists in aviation. Considering the human factor as the most important element in the air transport system (Luzik \& Akmaldinova, 2006), the results show the need for further research in the field of human factors in aviation, and more specifically, on those aspects directly related to the training and use of "new tools" to optimize crew coordination and performance.

Fortunately, nowadays the decrease in aviation accidents is astonishing. IATA Annual Review placed the global jet accident rate for 2014 at one major accident for every 4.4 million flights, "the lowest rate in the history of aviation" (IATA, 2015, p. 16). This optimistic data has been achieved, among many other factors, thanks to the standardization of the cockpit performances and the use of checklists. However, the aviation sector continues to present certain vulnerabilities, mainly the difficulty of coping with emergency situations for which it is not possible to create standardized procedures with the tools that the crews currently have. This research identifies these "black holes", showing empirically that not all situations are included in the checklists and proposing possible solutions based on training.

There are several trends for future research. In general terms, it would be interesting to advance the development of problem solving training programs that emphasize creativity, flexibility and divergent thinking, and to analyze the extent to which they really represent an "extra tool" for crews to deal with emergency situations not detailed by the checklists. Furthermore, it is important to determine whether the acquisition of such creativity skills will not interfere with the use of standardized procedures when their use is mandatory. That is, it is important to ensure that crews will be able to discern when to use the checklists and when to use their divergent thinking skills to get a solution.

\section{Acknowledgements}

The authors of this paper are grateful to the Spanish Air Force and, above all, to the pilots and flight engineers of the $43^{\text {rd }}$ Air Force Group for their generous and selfless collaboration on the present study.

All the opinions presented in this article belong to their authors and do not necessarily represent the opinion or official position of the Spanish Air Force, the Complutense University of Madrid or the University of León.

\section{Disclosure statement}

No financial, professional, or personal interests from other parties were reported by the authors.

\section{References}

Airbus. (2004). Flight operations briefing notes. Standard operating procedures. Normal checklists. Blagnac, France: Author.

Burke, C. S., Stagl, K. C., Salas, E., Pierce, L., \& Kendall, L. (2006). Understanding team adaptation: A conceptual analysis and model. Journal of Applied Psychology, 91(6), 11891207. https://doi.org/10.1037/0021-9010.91.6.1189

Degani, A., Heymann, M., \& Shafto, M. (1999). Formal aspects of procedures: The problem of sequential correctness. Proceedings of the Human Factors and Ergonomics Society Annual Meeting, 43(20), 1113-1117. https://doi.org/10.1177/154193129904302012

Degani, A., \& Wiener, E. L. (1990). Human factors of flightdeck checklists: The normal checklist (NASA Contractor Rep. 177549). Moffett Field, CA: NASA Ames Research Center.

Degani, A., \& Wiener, E. L. (1997). Procedures in complex systems: The airline cockpit. IEEE Transactions on Systems, Man, and Cybernetics, 27(3), 302-312. https://doi.org/10.1109/3468.568739

Dietz, A., Driskell, J., Sierra, M., Weaver, S., Driskell, T., \& Salas, E. (2017). Teamwork under stress. In E. Salas, R. Rico, \& J. Passmore (Eds.), The Psychology of Team Working and Collaborative Processes (pp. 297-315). West Sussex, UK: John Wiley \& Sons Ltd.

Gordon, S., Mendenhall, P., \& O'Connor, B. B. (2013). Beyond the checklist: what else health care can learn from aviation teamwork and safety. New York, NY: Cornell University Press.

Helmreich, R. L. (1999). Building safety on the three cultures of aviation. In Proceedings of the IATA Human Factors Seminar (pp. 39-43). Bangkok, Thailand, August 12, 1998. Retrieved from http://www.pacdeff.com/pdfs/3\%20Cultures\%20of\%20 Aviation\%20Helmreich.pdf

Helmreich, R. L. (2006). Red alert. Flight Safety Australia, 10(5), 24-31.

Helmreich, R. L., Klinect, J. R., Wilhelm, J. A., \& Jones, S. G. (1999). The line/LOS error checklist, Version 6.0: A checklist for human factors skills assessment, a log for off-normal events, and a worksheet for cockpit crew error management (Tech. Rep. No. 99-01). Austin, TX: University of Texas, Human Factors Research Project.

Helmreich, R. L., Merritt, A. C., \& Wilhelm, J. A. (1999). The evolution of Crew Resource Management training in commercial aviation. International Journal of Aviation Psychology, 9(1), 19-32. https://doi.org/10.1207/s15327108ijap0901_2

Helmreich, R. L., Wilhelm, J. A., Klinect, J. R., \& Merritt, A. C. (2001). Culture, error, and crew resource management. In E. Salas, C. A. Bowers, \& E. Edens (Eds.), Improving teamwork in organizations (pp. 305-331). Hillsdale, NJ: Erlbaum.

Hennessey, B. A., \& Amabile, T. A. (2010). Creativity. Annual Review of Psychology, 61, 569-598. https://doi.org/10.1146/annurev.psych.093008.100416

Heymann, M., Degani, A., \& Barshi, I. (2007). Generating procedures and recovery sequences: a formal approach. In Proceedings of the 14th international symposium on aviation psychology (pp. 252-257). Dayton, OH: Association for Aviation Psychology.

International Air Transport Association (2015). Annual Review. Miami, FL: Author. Retrieved from https://www.iata.org/ about/Documents/iata-annual-review-2015.pdf

Klinect, J. R., Murray, P., Merritt, A. C., \& Helmreich, R. L. (2003). Line operation safety audits (LOSA): Definition and 
operating characteristics. Proceedings of the 12th International Symposium on Aviation Psychology (pp. 663-668). Columbus, $\mathrm{OH}$ : The Ohio State University.

Luzik, E. V., \& Akmaldinova, A. N. (2006) Psychological aspects of ensuring flight safety in civil aviation, Aviation, 10(1), 25-35.

Marshall, D. (2010). Crew resource management: from patient safety to high reliability. Denver, CO: Safer Healthcare Partners.

Mosier, K. L., Palmer, E. A., \& Degani A. (1992). Electronic checklists: Implications for decision making. Proceeding of the Human Factors Society 36th Annual Meeting (pp. 7-11). Atlanta, GA: Human Factors Society.

Muñoz-Adánez, A. (2006). Métodos creativos para organizaciones. Madrid, España: Pirámide.

Muñoz-Marrón, D. (2018). Human Factors in Aviation: CRM (Crew Resource Management). Papeles del Psicólogo, 39(3), pp. 191-199. https://doi.org/10.23923/pap.psicol2018.2870

Norman, D. A. (1990). The design of everyday things. New York, NY: Doubleday.

Pettitt, M. A., \& Dunlap, J. H. (1997). Cockpit leadership and followership skills: Theoretical perspectives and training guidelines. Washington, DC: Federal Aviation Administration, AAR-100.

Rantz, W. G., Dickinson, A. M., Sinclair, G. A., \& Van Houten, R. (2009). The effect of feedback on the accuracy of checklist completion during instrument flight training. Journal of Applied Behavior Analysis, 42(3), 497-509.

https://doi.org/10.1901/jaba.2009.42-497

Reason J. (1990). Human error. New York, NY: Cambridge University Press. https://doi.org/10.1017/CBO9781139062367

Sculli, G. L., \& Sine, D. M. (2011). Soaring to success: taking crew resource management from the cockpit to the nursing unit. Danvers, MA: HCPro, a division of BLR.

Sine, D. M., \& Sculli, G. L. (2010). Just checking: using team briefings to improve patient safety. Healthbeat, 9(3), 4-6.

Stachowski, A. A., Kaplan, S. A., \& Waller, M. J. (2009). The benefits of flexible team interaction during crises. Journal of Applied Psychology, 94(6), 1536-1543. https://doi.org/10.1037/a0016903

Wieringa, D., Moore, C., \& Barnes, V. E. (1992). Procedure writing. Piscataway, NJ: IEEE Press.

Young-Xu, Y., Fore, A., Metcalf, A., Payne, K., Neily, J., \& Sculli, G. (2013). Using crew resource management and a 'Readand-Do Checklist' to reduce failure-to-rescue events on a step-down unit. AJN, American Journal of Nursing, 113(9), 51-57. https://doi.org/10.1097/01.NAJ.0000434178.06223.45

Zhou, J., \& Hoever, I. (2014). Research on workplace creativity: A review and redirection. Annual Review of Organizational Psychology and Organizational Behavior, 1, 333-359. https://doi.org/10.1146/annurev-orgpsych-031413-091226 